François Schweisguth Alain Israël

\section{ADRESSE}

F. Schweisguth: chargé de recherche au Cnrs. Institut Jacques-Monod, Cnrs université $\mathrm{Pa}$ ris VII, 75251 Paris Cedex 05. A. Israël: $d i$ recteur de recherche au Cnrs. Unité de biologie moléculaire de l'Expression génique, Cnrs URA 1149, Institut Pasteur, 75724 Paris Cedex 15, France.

\title{
Signalisation intercellulaire par le récepteur Notch: conservation de la drosophile aux mammifères
}

Au cours du développement embryonnaire des organismes multicellulaires complexes, un mécanisme nommé inhibition ou spécification latérale permet de contrôler dans l'espace et le temps la capacité des cellules de répondre à des signaux de différenciation. On a récemment mis à jour certains éléments des voies de transmission du signal par le récepteur Notch qui contrôle la différenciation de nombreux types cellulaires; par exemple, au cours de la neurogenèse chez la drosophile, l'activation du récepteur Notch stimule la transcription de gènes cibles et inhibe la différenciation, réglant ainsi le nombre de précurseurs neuroectodermiques qui choisiront un destin neuronal. La protéine $\mathrm{Su}(\mathrm{H})$, impliquée dans l'activité de transmission de ce signal, semble capable de s'associer à la région intracellulaire de Notch. C'est un facteur de transcription qui se lie à certaines séquences localisées dans les promoteurs de plusieurs des gènes cibles de Notch. La voie de signalisation de Notch semble conservée chez les mammifères.

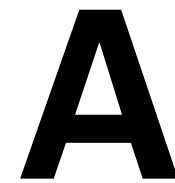

$\mathrm{u}$ cours du développement embryonnaire, le destin d'une cellule est déterminé par son lignage Ainsi, de nombreux signaux extracellulaires règlent la capacité des cellules qui les reçoivent de se diviser, de migrer ou encore de se différencier en un type cellulaire donné. La plupart de ces signaux sont dits «ins- tructifs » car ils déterminent le devenir de la cellule réceptrice. Des études effectuées chez le nématode et la drosophile ont permis d'identifier un second type de signal, appelé inhibition latérale ou spécification latérale $[1,2]$. Ces signaux ne semblent pas avoir de valeur «instructive", dans la mesure où ils ne confèrent pas une identité cellulaire particulière, mais servent plutôt 


\section{RÉFÉRENCES}

1. Greenwald I, Rubin GM. Making a difference: the role of cell-cell interactions in establishing separate identities for equivalent cells. Cell 1992; 68: 271-81.

2. Sternberg PW. Falling off the knife edge. Current Biology 1993; 3 : 763-5.

3. Wilkinson HA, Fitzgerald K, Greenwald I. Reciprocal changes in expression of the receptor Lin-12 and its ligand Lag-2 prior to commitment in a C. elegans cell fate decision. Cell 1994; 79 : 1187-98.

4. Artavanis-Tsakonas S, Matsuno K, Fortini ME. Notch signaling. Science 1995; 268 : 22532

5. Campos-Ortega JA. Early neurogenesis in Drosophila Melanogaster. In: Bate M, Martinez-Arias A eds. The Development of Drosophila Melanogaster, vol. 2, Cold Spring Harbor Laboratory Press, 1993: 1091-129.

6. Ghysen A, Dambly-Chaudière C, Jan LY, Jan YN. Cell interactions and gene interactions in peripheral neurogenesis. Genes Dev $1993 ; 7: 723-33$.

7. Fortini ME, Artavanis TS. Notch : neurogenesis is only part of the picture. Cell 1993; $75: 1245-7$.

8. Greenwald I. Structure/function studies of Lin-12/Notch proteins. Curr Opin Genet Dev 1994; 4: 556-62.

9. Lieber T, Kidd S, Alcamo E, Corbin V, Young MW. Antineurogenic phenotypes induced by truncated Notch proteins indicate a role in signal transduction and may point to a novel function for Notch in nuclei. Genes Dev 1993; 7: 1949-65.

10. Rebay I, Fehon RG, Artavanis-Tsakonas S. Specific truncations of Drosophila Notch define dominant activated and dominant negative forms of the receptor. Cell 1993; $74: 319-29$.

11. Matsuno K, Diederich RJ, Go MJ, Blaumuller CM, Artavanis-Tsakonas S. Deltex acts as a positive regulator of Notch signaling through interactions with the Notch ankyrin repeats. Development 1995; 121 : 2633-44.

12. Fortini ME, Artavanis-Tsakonas S. The Suppressor of Hairless protein participates in Notch receptor signaling. Cetl 1994; 79 . 273-82.

13. Lecourtois M, Schweisguth F. The neurogenic Suppressor of Hairless DNA-binding protein mediates the transcriptional activation of the Enhancer of Split Complex genes triggered by Notch signaling. Genes à établir et stabiliser une différence de destinée au sein d'une population de cellules équipotentes. Nous résumerons tout d'abord les données relatives au rôle et au mécanisme de transduction des signaux d'inhibition latérale au cours du développement des invertébrés, puis nous présenterons les résultats obtenus chez les mammifères qui suggèrent une conservation à la fois structurale et fonctionnelle du mécanisme d'inhibition latérale.

\section{Un exemple d'inhibition latérale chez le nématode}

L'inhibition latérale est un processus dynamique par lequel des cellules adjacentes équipotentes établissent de manière stable, par l'échange réciproque de signaux inhibiteurs, une différence d'identité. L'exemple le plus simple, et l'un des mieux étudiés, est donné par la détermination de la cellule ancre chez le nématode [1] (figure 1). Deux cellules voisines, dénotées Z1.ppp et Z4.aaa, adoptent de manière équiprobable la destinée soit de cellule ancre (AC), soit de cellule précurseur de la vulve (VU): dans $50 \%$ des individus, Z1.ppp devient « $\mathrm{AC}$ » et $\mathrm{Z} 4$.aaa « $\mathrm{VU}$ », et dans les $50 \%$ autres, c'est l'inverse. En revanche, si l'une quelconque des cellules Z1.ppp ou Z4.aaa est éliminée expérimentalement, la cellule restante devient toujours «AC». Cette observation suggère qu'un signal inhibiteur émis par la future cellule «AC » empêche sa voisine d'adopter la même destinée. Des données génétiques et moléculaires indiquent que les produits des gènes $\mathrm{Lin}$-12 et Lag-2 agissent respectivement comme récepteur et ligand lors de la transmission de ce signal d'inhibition latérale. Ces gènes sont exprimés tous deux dans les cellules Z1.ppp et Z4.aaa avant leur détermination. La synthèse du récepteur Lin-12 est progressivement restreinte à la cellule «VU» (qui reçoit le signal inhibiteur) et celle du ligand Lag-2 à la cellule «AC » (qui émet le signal inhibiteur). Comment s'établit cette différence d'expression et de destinée? Il a été récemment montré que l'activation du récepteur Lin-12 à la surface cellulaire stimule la transcription du gène Lin-12 [3]. Ainsi, la cellule qui reçoit initialement un peu

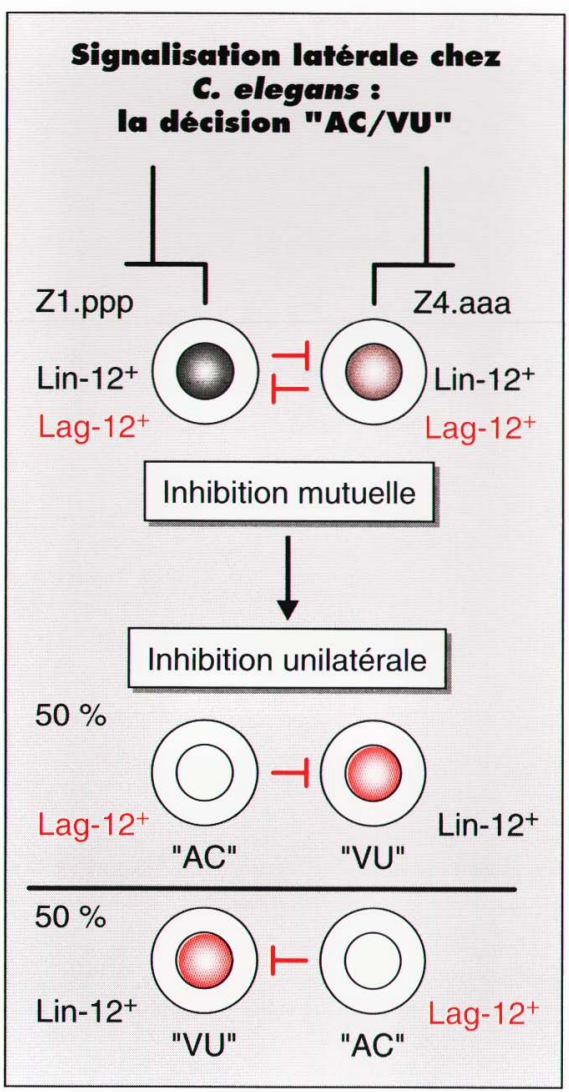

Figure 1. Exemple de signalisation latérale: la décision $A C / V U$ chez $C$. elegans. Deux cellules voisines, Z1.ppp et Z4.aaa ont, au départ, une chance identique de devenir cellule ancre AC ou cellule de la vulve VU. Les produits de deux gènes Lin-12 et Lag-2 agissent respectivement comme récepteur et ligand lors de la transmission d'un signal d'inhibition latérale et sont synthétisés tous deux dans les cellules Z1.ppp et Z4.aaa avant leur détermination; puis la synthèse du récepteur Lin-12 va progressivement se restreindre à la cellule VU et celle du ligand Lag-2 à la cellule AC. Cette différence d'expression, autoamplifiée, est liée au fait que l'activation du récepteur Lin-12 à la surface de la cellule stimule la transcription du gène Lin-12.

plus de signal inhibiteur voit sa capacité de recevoir le signal augmentée. Ce mécanisme d'autorégulation permet d'amplifier une différence initiale faible et aléatoire entre Z1.ppp et Z4.aaa pour aboutir à une différence irréversible entre une cellule " $\mathrm{AC}$ » inhibitrice et une cellule «VU» réceptrice. Dans cet exemple, l'inhibi- 
tion latérale permet d'établir et de

\section{RÉFÉRENCES}

14. Schweisguth F. Suppressor of Hairless is required for signal reception during lateral inhibition in the Drosophila pupal notum. Development 1995 ; 121 ; 1875-84.

15. Schweisguth F, Posakony JW. Suppressor of Hairless, the Drosophila homolog of the mouse recombination signal-binding protein gene, controls sensory organ cell fates. Cell 1992; 69 : 1199-212.

16. Schweisguth F, Posakony JW. Antagonistic activities of Suppressor of Hairless and Hairless control alternative cell fates in the Drosophila adult epidermis. Development 1994; 120 : 1433-41.

17. Schweisguth F, Gho M, Lecourtois M. Control of cell fate choices by lateral signaling in the adult peripheral nervous system of Drosophila melanogaster. Dev Genetics 1996 , sous presse.

18. Bailey AM, Posakony JW. Suppressor of Hairless directly activates transcription of Enhancer of Split Complex genes in response to Notch receptor activity. Genes Dev $1995 ; 9$ : 2609-22.

19. Brou C, Logeat F, Lecourtois M, Vandekerckhove I, Kourilsky P, Schweisguth F, Israel $A$. Inhibition of the DNA-binding activity of Drosophila Suppressor of Hairless and of its human homolog, KBF2/RBP-Jא, by direct protein-protein interaction with Drosophila Hairless. Gene Dev 1994; 8: 2491-503.

20. Franco del Amo F, Smith DE, Swiatek PI, Gendron-Maguire M, Greenspan RJ, McMahon AP, Gridley T. Expression pattern of Motch, a mouse homolog of Drosophila Notch, suggests an important role in early postimplantation mouse development. Development 1992 ; 115 : 737-44.

21. Lardelli M, Dahlstrand J, Lendahl U. The novel Notch homologue mouse Notch 3 lacks specific epidermal growth factor-repeats and is expressed in proliferating neuroepithelium. Mech Dev 1994; 46: 123-36.

22. Reaume AG, Conlon RA, Zirngibl R, Yamaguchi TP, Rossant J. Expression analysis of a Notch homologue in the mouse embryo. Dev Biol 1992; 154: 377-87.

23. Weinmaster G, Roberts VJ, Lemke G. Notch 2 : a second mammalian Notch gene. Development 1992; 116: 931-41.

24. Swiatek PJ, Lindsell CE, Delamo FF, Weinmaster G, Gridley T. Notch1 is essential for postimplantation development in mice. Genes Dev 1994; 8: 707-19.

$\mathrm{m} / \mathrm{s} n^{\circ} 2$, vol. 12 , féurier 96 maintenir une différence d'identité entre deux cellules équipotentes.

\section{Transduction du signal d'inhibition latérale chez la drosophile}

\section{Structure et fonction du récepteur Notch}

kyrine (figure 2). Lag-2 appartient à une autre famille de protéines transmembranaires, la famille DSL (DeltaSerrate-Lag-2). Delta et Serrate sont deux protéines de drosophile capables d'interagir avec Notch dans l'espace extracellulaire. Il apparaît donc que la structure de la moléculesignal et celle du récepteur ont été conservées entre le nématode et la drosophile.

Lin-12 est un membre distant de la Le rôle de Notch chez la drosophile famille des récepteurs transmembra- a été particulièrement étudié lors de naires de type Notch [4] (figure 2). La protéine Notch de drosophile est une protéine d'à peu près 300 kDa, constituée de 36 répétitions du motif EGF (epidermal growth factor) et 3 répétitions du type Notch/Lin12, riches en cystéines, dans la partie extracellulaire, un domaine transmembranaire et une large région intracellulaire comportant 6 répétitions de type anla formation du système nerveux [5, 6]. Au cours de la neurogenèse chez l'embryon, un territoire composé d'environ 2000 cellules, le neuroectoderme, donne naissance à environ 500 précurseurs du système nerveux central, les neuroblastes, et 1500 précurseurs de l'épiderme larvaire, les dermoblastes. Les cellules du neuroectoderme se voient conférer la ca-

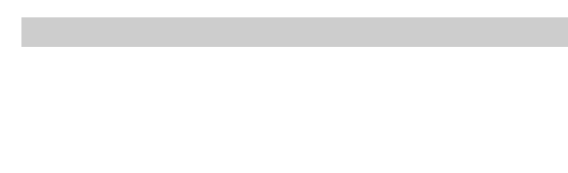

Figure 2. Structure schématique des récepteurs Notch et de leurs ligands. La protéine Notch (la molécule de drosophile est représentée ici) contient 36 répétitions EGF ainsi que 3 répétitions Lin-12/Notch dans la partie extracellulaire, et 6 répétitions cdc10/ankyrine dans sa partie intracellulaire. La protéine Notch-1 isolée chez I'homme, le rat et la souris, possède également 36 répétitions EGF. Les protéines homologues de $C$. elegans, Glp-1 and Lin-12, sont légèrement plus divergentes et présentent respectivement 11 et 14 répétitions EGF. La protéine Delta de drosophile est formée d'une courte région intracellulaire dont la fonction est inconnue et, dans sa partie extracellulaire, de 9 répétitions EGF et d'un domaine $N$-terminal appelé DSL (pour DeltaSerrate-Lag-2) responsable de l'interaction avec Notch. Les protéines Delta de xénope, poulet et souris, contiennent 8 répétitions EGF. La protéine Serrate de drosophile comporte 14 répétitions EGF, dont 3 sont interrompues par des insertions; I'homologue probable chez le rat, Jagged, comporte 16 répétitions EGF. Comme pour Notch, les protéines de $C$. elegans (Apx-1 et Lag-2) sont plus divergentes et il est difficile de les classer comme des homologues de Delta ou de Serrate. Chez la drosophile, les répétitions EGF 11 et 12 de Notch sont responsables de I'interaction avec Delta et Serrate. 


\section{RÉFÉRENCES}

25. Conlon RA, Reaume AG, Rossant J. Notch 1 is required for the coordinate segmentation of somites. Development 1995; 121 : $1533-45$

26. Kopan R, Nye JS, Weintraub H. The intracellular domain of mouse Notch: a constitutively activated repressor of myogenesis directed at the basic helix-loop-helix region of MyoD. Development 1994 ; 120 : 2385-96.

27. Nye JS, Kopan R, Axel R. An activated Notch suppresses neurogenesis and myogenesis but not gliogenesis in mammalian cells. Development 1994; 120 : 2421-30.

28. Coffman CR, Skoglund P, Harris WA, Kintner CR. Expression of an extracellular deletion of Xotch diverts cell fate in xenopus embryo. Cell 1993; 73: 659-71.

29. Ellisen LW, Bird J, West DC, Soreng AL, Reynolds TC, Smith SD, Sklar J. TAN-1, the human homolog of the Drosophila notch gene, is broken by chromosomal translocations in $\mathrm{T}$ lymphoblastic neoplasms. Cell $1991 ; 66$ : 649-61.

30. Lindsell CE, Shawber CJ, Boulter J, Weinmaster G. Jagged : a mammalian ligand that activates Notch1. Cell 1995; 80 : 909-17.

31. Matsunami N, Hamaguchi Y, Yamamoto Y, Kuze K, Kangawa K, Matsuo H, Kawaichi M, Honp T. A protein binding to the J kappa recombination sequence of immunoglobulin genes contains a sequence related to the integrase motif. Nature 1989 ; 342 : 934 7 .

32. Tun T, Hamaguchi Y, Matsunami N, Furukawa T, Honp T, Kawaichi M. Recognition sequence of a highly conserved DNA binding protein RBP-Jkappa. Nucleic Acids Res 1994; 22 : 965-71.

33. Israel A, Yano O, Logeat F, Kieran M, Kourilsky P. Two purified factors bind to the same sequence in the enhancer of mouse MHC class I genes: one of them is a positive regulator induced upon differentiation of teratocarcinoma cells. Nucleic Acids Res 1989; 17 : 5245-57.

34. Henkel T, Ling PD, Hayward D, Peterson MG. Mediation of Epstein-Barr virus EBNA2 transactivation by recombination signal-binding protein Jkappa. Science 1994 ; 265: $92-5$

35. Ling PD, Hsieh IJ, Ruf IK, Rawlins DR, Hayward SD. EBNA-2 upregulation of Epstein-Barr virus latency promoters and the cellular CD23 promoter utilizes a common targeting intermediate, CBF1. I Virol 1994; pacité de se différencier en neuroblaste par l'expression d'une classe de gènes appelés gènes proneuraux. Les principaux gènes proneuraux appartiennent au complexe achaete-scute et codent pour des facteurs de transcription. Des groupes de 5 à 6 cellules expriment de manière transitoire ces gènes. Au sein de ce groupe de cellules équipotentes, appelé groupe proneural, une seule cellule donnera naissance à un neuroblaste. Les autres cellules du groupe proneural restent indifférenciées et pourront faire partie un peu plus tard d'un second groupe proneural. Ainsi, il semble que toutes les cellules du neuroectoderme peuvent adopter l'une ou l'autre de ces deux destinées cellulaires, selon une séquence spatiotemporelle complexe résultant du profil d'expression des gènes proneuraux dans le neuroectoderme.

L'établissement d'une distinction stable entre neuroblaste et dermoblaste requiert l'activation de Notch par Delta. Dans un embryon mutant pour Notch ou pour Delta, toutes les cellules du neuro-ectoderme se différencient en neuroblaste. Cela suggère que l'activation de Notch par Delta empêche les cellules du neuroectoderme de se différencier en neuroblaste. Les cellules dont le récepteur Notch est activé restent alors insérées au sein du neuroectoderme, se divisent et conservent leur caractère pluripotent. Ainsi, la fonction de Notch chez la drosophile serait de bloquer la différenciation cellulaire et de maintenir la capacité des cellules recevant le signal transmis par Notch de recevoir ultérieurement d'autres signaux de différenciation [7]. L'inhibition latérale permet donc de régler dans l'espace et le temps la capacité des cellules de répondre à des signaux de différenciation. Chez la drosophile, ce mécanisme d'inhibition latérale relayé par Notch contrôle la différenciation de très nombreux types cellulaires tout au long du développement (tels que les cellules précurseurs des muscles larvaires, les cellules de l'intestin moyen, du système nerveux périphérique, de l'œil composé, de la chambre ovarienne...).

L'analyse génétique de la formation du système nerveux a permis une dissection moléculaire du signal d'inhibition latérale. Cette analyse a porté, d'une part, sur les rapports entre structure et fonction du récepteur Notch, d'autre part, sur les différentes protéines impliquées dans la transmission intracellulaire du signal d'activation du récepteur. L'analyse fonctionnelle des différents domaines de Notch est en cours d'étude dans plusieurs laboratoires [8]. Ces études reposent sur la réalisation de molécules Notch dont certaines régions spécifiques ont été délétées et sur l'analyse de l'activité de ces molécules in vivo après transgenèse. Deux classes de constructions se sont avérées particulièrement instructives. Tout d'abord, les versions de Notch dont le domaine intracellulaire a été délété (c'est-à-dire conservant la partie extracellulaire du récepteur ainsi que le domaine transmembranaire) se comportent généralement de manière «dominante négative». En d'autres termes, l'expression de cette classe de molécules dans un embryon ou une mouche sauvage conduit à des anomalies identiques à celles observées lorsque l'activité de Notch est réduite, incluant, entre autres, une hypertrophie du système nerveux. Cela suggère que ces molécules sont capables d'interférer avec la fonction du récepteur Notch endogène, probablement en fixant le ligand Delta sans pour autant activer la voie de signalisation Notch. L'expression de ces molécules limiterait ainsi la quantité de ligand disponible pour une interaction avec les récepteurs Notch endogènes. Ce test d'activité dominante négative permet alors de définir au sein du domaine intracellulaire la région nécessaire à l'activation de la voie de signalisation Notch. Une analyse par délétion de cette région (longue d'environ 1000 acides aminés) indique que les répétitions du motif ankyrine sont nécessaires pour cet effet dominant négatif. Il est important de noter que, selon les études, d'autres domaines intracellulaires de Notch semblent nécessaires à la transmission du signal (voir [9, 10], par exemple). La seconde classe de constructions correspond à des versions de Notch dont la partie extracellulaire a été délétée, et qui conservent, ou non, le domaine transmembranaire. Ces molécules sont dites «dominantes actives» car leur expression confère des phénotypes d'activation constitutive de la 
voie de signalisation Notch. Par exemple, l'expression de telles formes tronquées bloque la délamination et la détermination des neuroblastes. Il en résulte une hypotrophie du système nerveux. L'analyse fonctionnelle de ces versions tronquées de Notch indique à nouveau que la partie intracellulaire de ce récepteur, avec ses répétitions du motif ankyrine, est suffisante pour constituer une molécule Notch activée [8]. Ainsi, Notch peut être activé, soit par la liaison de son ligand Delta, soit de manière artificielle en délétant son domaine extracellulaire. On en déduit que la liaison de Delta à Notch lèverait l'inhibition que fait normalement peser le domaine extracellulaire sur l'activité intrinsèque du domaine intracellulaire. Les répétitions du domaine ankyrine pourraient former l'ossature d'une surface d'interaction protéine-protéine. Cela suggère que l'activation initiale $\mathrm{du}$ récepteur Notch au niveau de la membrane plasmique pourrait être relayée par une (ou plusieurs) protéine(s) se liant à ces répétitions.

\section{Rôle de Suppressor of Hairless dans la voie de signalisation Notch}

Deux protéines ont été identifiées à ce jour qui interagissent directement avec les répétitions du motif ankyrine de Notch (en test double hybride chez la levure et par co-localisation dans des cellules transfectées): une protéine cytoplasmique, Deltex, identifiée du fait des interactions génétiques entre deltex et Notch, qui semblent jouer un rôle facilitateur encore mal défini lors de la transduction du signal transmis par Notch [11]; et Suppressor of Hairless [Su(H) ] [12]. Un grand nombre de données génétiques et moléculaires indiquent que $\mathrm{Su}(\mathrm{H})$ joue un rôle central dans la transduction du signal transmis par Notch. Tout d'abord, la mutation du gène $S u(H)$ entraîne des anomalies de développement identiques à celles observées dans un mutant Notch. En particulier, $S u(H)$ est requis pour la formation du système nerveux de la larve (au cours de l'embryogenèse) et de l'adulte (lors de la métamorphose) [13-15]. Dans un embryon mutant pour $S u(H)$, toutes les cellules du neuroectoderme se différencient en neuroblaste. Il en résulte une hypertrophie du système nerveux. Réciproquement, la surexpression de $S u(H)$ conduit à un phénotype de même type que celui dû à l'activation déréglée de Notch [16]. De plus, lors de la formation des organes sensoriels, l'activité du gène $S u(H)$ est requise dans les cellules qui reçoivent le signal d'inhibition (plutôt que dans celles qui le produisent), indiquant que $\mathrm{Su}(\mathrm{H})$ participe à la transmission de ce signal (plutôt qu'à la production du signal lui-même) [14]. Enfin, les interactions génétiques détectées entre $\mathrm{Su}(H)$ et Notch suggèrent que $\mathrm{Su}(\mathrm{H})$ participe de manière directe à la voie de transmission du signal Notch [12, 17].

Sur le plan moléculaire, la protéine $\mathrm{Su}(\mathrm{H})$ est un facteur de transcription se liant aux séquences régulatrices de plusieurs gènes dont l'expression est induite en réponse à l'activation du récepteur Notch: c'est le cas des gènes du complexe Enhancer of split $[E(s p l)]$ lors de la neurogenèse [13, 18]. Ce complexe génétique est composé de huit gènes fonctionnellement redondants et exprimés de manière semblable dans l'embryon lors de la formation du système nerveux. Sept de ces gènes codent pour des protéines se liant à l'ADN de la famille hélice-boucle-hélice (HLH), du nom de la structure du motif de liaison à l'ADN. Ces protéines E(spl) semblent intervenir de deux façons complémentaires pour réprimer la transcription des gènes proneuraux achaete-scute qui gouvernent la différenciation neurale: d'une part, elles se lieraient aux séquences régulatrices de ces gènes et y recruteraient, par interaction protéine-protéine directe, un répresseur de la transcription appelé Groucho; d'autre part, ces protéines $\mathrm{E}(\mathrm{spl})$ seraient capables de se lier aux facteurs de transcription, également de la famille héliceboucle-hélice, codés par ces gènes proneuraux pour bloquer leur activité de liaison à l'ADN.

$\mathrm{Su}(\mathrm{H})$ est en outre la cible de l'activité du gène Hairless qui, génétiquement, se comporte comme un inhibiteur spécifique de la transduction du signal transmis par Notch. En effet, la protéine Hairless est capable de se lier in vitro à la protéine $\mathrm{Su}(\mathrm{H})$, l'empêchant ainsi de se fixer à l'ADN et donc de régler la transcription des gènes cibles de $\mathrm{Su}(\mathrm{H})$ et du signal transmis par Notch [19]. Ces données suggèrent un modèle linéaire simple (figure 3): l'activation du récepteur Notch par Delta «active» le facteur de transcription $\mathrm{Su}(\mathrm{H})$ qui stimule la transcription des gènescibles du signal transmis par Notch. Ce modèle moléculaire ne précise pas comment Notch «activerait» $\mathrm{Su}(\mathrm{H})$ (voir les deux modèles décrits ci-dessous, figure 3).

L'interaction entre une protéine transmembranaire et un facteur de transcription nucléaire pose évidemment la question du compartiment cellulaire où a lieu cette interaction. Cette question centrale n'a pas encore été tranchée. Une possibilité est que $\mathrm{Su}(\mathrm{H})$ interagisse avec le domaine intracellulaire de Notch dans le compartiment sous-membranaire. Cette hypothèse est en accord avec les résultats d'expériences de transfection cellulaire montrant que $\mathrm{Su}(\mathrm{H})$ est co-localisé avec Notch dans le cytoplasme en l'absence du ligand Delta [12]. En revanche, l'activation de Notch par Delta semble empêcher l'interaction entre $\mathrm{Su}(\mathrm{H})$ et Notch à l'intérieur de la cellule, entraînant la localisation de $\mathrm{Su}(\mathrm{H})$ dans le noyau [12]. A la différence de $\mathrm{Su}(\mathrm{H})$, deltex semble recruté par Notch (en interagissant avec ces mêmes répétitions ankyrines) lorsque Delta se lie à Notch à l'extérieur de la cellule [11]. En accord avec ces données, il a été montré que la liaison de $\mathrm{Su}(\mathrm{H})$ à Notch exclut celle de deltex à Notch [11]. Ces données suggèrent le modèle suivant [4]: $\mathrm{Su}(\mathrm{H})$ serait lié au récepteur Notch non activé. L'activation de Notch par Delta entraînerait la libération de $\mathrm{Su}(\mathrm{H})$ et la liaison de deltex à Notch. $\mathrm{Su}(\mathrm{H})$ serait alors importé dans le noyau où il activerait la transcription des gènes du complexe $\mathrm{E}(\mathrm{spl})$, cibles génomiques du signal transmis par Notch. Dans ce modèle, la translocation nucléaire de $\mathrm{Su}(\mathrm{H})$ est ainsi l'événement central de cette voie de signalisation. Il sera donc important de déterminer avec précision la localisation sub-cellulaire de $\mathrm{Su}(\mathrm{H})$ in vivo lors de la neurogenèse, afin d'identifier d'éventuels changements de localisation nucléo-cytoplasmique lors de la transduction du signal transmis par Notch.

On peut également envisager que l'interaction entre $\mathrm{Su}(\mathrm{H})$ et le domaine intracellulaire de Notch ait 


\section{RÉFÉRENCES}

36. Waltzer L, Logeat F, Brou C, Israel A, Sergeant A, Manet E. The human Jkappa recombination signal sequence binding protein (RBP-Jk) targets the Epstein-Barr virus EBNA2 protein to its DNA responsive elements. EMBO J 1994 ; 13 ; 5633-8.

37. Hsieh JD, Hayward D. Masking of the CBF1/RBP-Jk transcriptional repression domain by Epstein-Barr virus EBNA2. Science $1995 ; 268: 560-3$.

38. Sasai Y, Kageyama R, Tagawa Y, Shigemoto R, Naganishi S. Two mammalian helix-loop-helix factors structurally related to Drosophila Hairy and Enhancer of split. Genes Dev 1992; 6: 2620-34.

39. Johnson JE, Birren SJ, Anderson DJ. Two rat homologues of Drosophila achaetescute specifically expressed in neuronal precursors. Nature 1990; 346: 858-61.

40. Kopan R, Schroeter EH, Nye JS, Weintraub H. Signal transduction by activated mNotch : importance of proteolytic processing and its regulation by the extracellular domain. Proc Natl Acad Sci USA 1996, sous presse.

41. Jarriault S, Brou C, Logeat F, Schroeter E, Kopan R, Israel A. Signalling downstream of activated mammalian Notch. Nature $1995 ; 377: 355-8$

42. Oka C, Nakano T, Wakeham A, de la Pompa JL, Mori C, Sakai T. Okazaki S, Kawaicho M, Shiota K, Mak TW, Honjo T. Disruption of the mouse $R B P-J k$ gene results in early embryonic death. Development 1995; 121: 3291-301.

43. Nye JS, Kopan R. Vertebrate ligands for Notch. Curr Biol 1995 ; 5 : 966-9.

44. Chitnis A, Henrique D, Lewis J, Ish-Horowicz D, Kintner C. Primary neurogenesis in Xenopus embryos regulated by a homologue of the Drosophila neurogenic gene Delta. Nature 1995 ; 375 : 761-6.

45. Henrique D, Adam J, Myat A, Chitnis A, Lewis J, Ish-Horowicz D. Expression of a Delta homologue in prospective neurons in the chick. Nature 1995; 375: 787-90.

46. Bettenhausen B, Hrabe de Angelis M, Simon D, Guenet JL, Gossler A. Transient and restricted expression during mouse embryogenesis of Dll1, a murine gene closely related to Drosophila Delta. Development $1995 ; 121: 2407-18$.

47. Austin CP, Feldman DE, Ida J, Cepko C. Vertebrate retina ganglion cells are selected from competent progenitors by the action

\section{• Modèle "importation nucléaire" :}

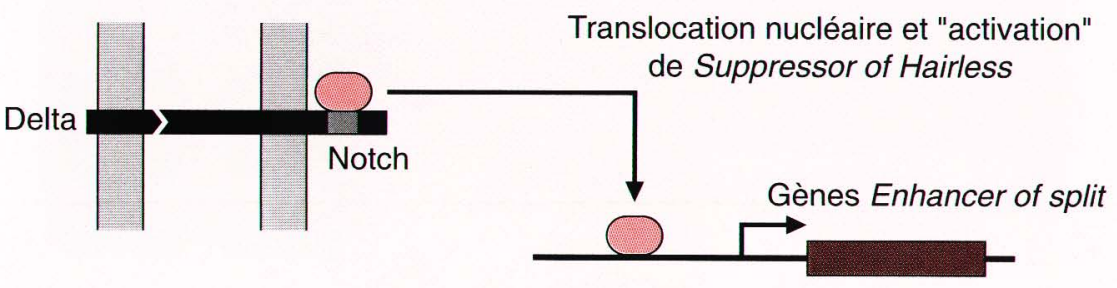

B - Modèle "coupure/libération du coactiveur" :

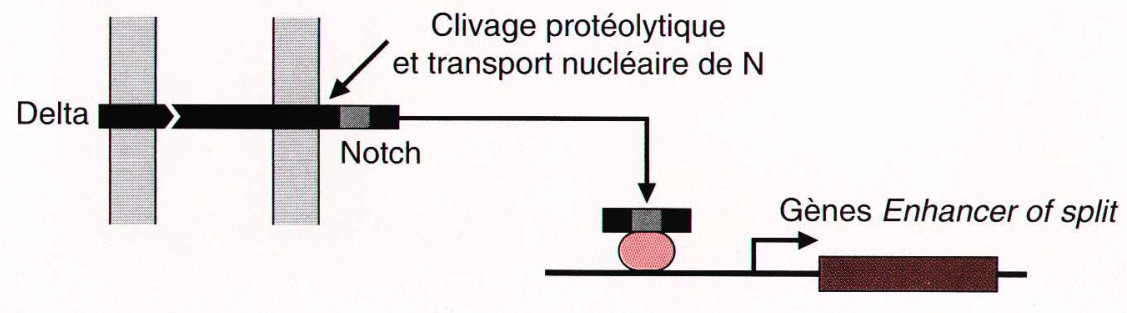

Figure 3. Deux modèles d'activation de la transcription à la suite de l'interaction de Notch avec son ligand Delta. Deux modèles peuvent rendre compte de l'activation de la transcription résultant de l'activation de Notch. A. La protéine Suppressor of Hairless, $S u(H)$, liée au domaine cytoplasmique de Notch non activé, est libérée de cette interaction après activation de Notch et transférée dans le noyau où elle peut exercer son rôle de facteur de transcription des gènes du complexe $E(s p l)$, les cibles génomiques du signal transmis par Notch. B. La protéine $S u(H)$ est localisée dans le noyau et le domaine cytoplasmique de Notch, mûri par protéolyse lors de l'activation du récepteur Notch, I'y rejoint; là elle "active" les propriétés pro-transcriptionnelles de $\mathrm{Su}(\mathrm{H})$.

lieu dans le noyau. Cette hypothèse, qui implique la production du domaine intracellulaire de Notch par clivage protéolytique, découle notamment de données obtenues récemment chez les mammifères (voir cidessous), ainsi que de l'observation de la localisation nucléaire des versions dominantes actives de Notch dont la partie extracellulaire et le domaine transmembranaire ont été délétés. En outre, l'analyse par la technique de Western blot des différents polypeptides reconnus par un anticorps dirigé contre la partie intracellulaire du récepteur suggère que Notch est mûri pour donner naissance à un polypeptide d'environ $100 \mathrm{kDa}$. Enfin, la séquence des signaux de localisation nucléaire présents dans la partie intracellulaire de Notch a été conservée au cours de l'évolution. La fonction éventuelle du fragment intracellulaire de Notch dans le noyau est discutée ci-dessous. Toutefois, in vivo, les molécules Notch endogènes ne sont détectées qu'à la membrane et dans des vésicules cytoplasmiques [4].

\section{La voie de signalisation Notch-Su(H) chez les mammifères}

Les informations concernant la fonction de Notch chez les mammifères sont relativement peu abondantes et fondées essentiellement sur le profil d'expression au cours du développement embryonnaire des différents récepteurs Notch et de leur ligands présumés, sur quelques données fonctionnelles obtenues dans des systèmes cellulaires ex vivo ou par injection chez le xénope, et sur les données génétiques préliminaires provenant de l'étude de souris mutées pour Notch-1 et $\mathrm{Su}(\mathrm{H})$. En outre, un certain nombre de données 
concernant la fonction de la protéine $\mathrm{Su}(\mathrm{H})$ ont été obtenues chez l'homme dans un contexte indépendant de la cascade Notch.

\section{Les protéines Notch chez les mammifères}

Trois gènes Notch ont été clonés chez les mammifères (Notch 1, 2 et 3) [2023]. La structure des trois protéines pour lesquelles ces gènes codent est identique à celle de la protéine de drosophile. Les profils d'expression de ces gènes au cours du développement sont distincts, suggérant des activités non redondantes. Le profil d'expression de Notch 1 murin, qui a été le plus étudié, indique un rôle probable dans la formation du mésoderme, des somites et du système nerveux. L'ARNm de Notch 1 est détecté dans le mésoderme dès le jour 7,5 du développement embryonnaire; à 9,5 jours, le site majeur d'expression est le neuroépithélium, ainsi que les tissus dérivés de la crête neurale. Une source d'informations supplémentaire résulte de l'inactivation par recombinaison homologue du gène Notch 1 murin [24, 25] : les embryons présentant une inactivation des deux allèles de Notch 1 se développent normalement jusqu'au jour 9 , mais meurent au jour 10. Une analyse histologique des embryons indique une importante mort cellulaire, plus spécialement dans les régions du système nerveux central et périphérique.

L'ensemble de ces observations n'apporte guère d'informations concernant la fonction de Notch chez les mammifères. En revanche, l'utilisation de lignées cellulaires qui permet d'induire la différenciation vers un type tissulaire donné a apporté des résultats qui sont plutôt en accord avec ce qu'on connait de la fonction de Notch chez la drosophile. L'introduction dans des myoblastes C2 d'un mutant de Notch-1 contenant uniquement la région intracellulaire (qui se comporte chez la drosophile comme une forme constitutivement active de Notch) bloque leur différenciation en myotubes [26]. Dans des cellules de tératocarcinome P19, l'introduction de cette même forme de Notch-1 bloque la différenciation en neurones ou en muscle, mais pas en cellules gliales [27]. Chez le xénope, la production d'une forme acti$\mathrm{m} / \mathrm{s} n^{\circ} 2$, vol. 12, féurier 96 vée de façon constitutive de XNotch cause la perte des structures dorsales de même qu'une hypertrophie neurale et mésodermique [28]. Ces phénotypes suggèrent que la forme activée de XNotch bloque ou retarde la différenciation de ces cellules, et les maintient dans un état prolifératif. Ces données sont à rapprocher du fait que Notch 1 a été cloné chez l'homme en tant qu'oncogène [29], de même que chez la souris un autre membre plus distant de la famille, int-3. Dans les deux cas l'expression non contrôlée d'un ARN messager codant pour la région intracellulaire de la molécule semble interférer avec la différenciation cellulaire normale et provoquer un phénotype transformé. Plus récemment il a été montré que la surreprésentation à la surface de fibroblastes de souris d'un ligand potentiel de Notch, Jagged (analogue probable du ligand Serrate de drosophile) bloque la différenciation de myoblastes C2 transfectés par Notch 1 et mis en contact avec ces fibroblastes [30]. Ces divers résultats semblent indiquer que chez les mammifères, Notch joue un rôle d'inhibiteur de différenciation activé par un signal extracellulaire, une situation identique à ce qui a été postulé chez la drosophile.

\section{La protéine $\mathrm{Su}(\mathrm{H})$ chez les mammifères}

L'orthologue de $S u(H)$ chez les mammifères a été cloné par plusieurs groupes dans des conditions très différentes, et indépendantes de la voie de signalisation Notch. Le groupe de T. Honjo a purifié en 1989 une protéine de 55-60 kD se liant à un site contenant la séquence GTGGGAA, et a cloné le gène correspondant appelé $R B P-/ \mathrm{k}$ [31, 32]. Parallèlement, l'un de nous a cloné l'ADNc codant pour une protéine se liant aux sites de fixation du facteur de transcription NF-кB [33]. Nous avons nommé cette protéine KBF2 et avons déterminé qu'elle se fixait à un demi-site $\mathrm{NF}-\kappa \mathrm{B}$, qui représente une séquence compatible avec le consensus GTGGGAA. Cette protéine semble dépourvue d'activité transcriptionnelle. La question de savoir si la fixation de KBF2 aux sites NFKB a une réelle signification physiologique n'a toujours pas reçu de réponse nette. Fina- lement divers groupes étudiant le mode d'action de la protéine EBNA2, qui est un activateur transcriptionnel codé par le virus d'Epstein-Barr (EBV) et ne se liant pas à l'ADN, ont montré que la protéine KBF2/RBP-JK servait de point d'ancrage à l'ADN pour EBNA2, lui permettant ainsi d'activer ses gènes cibles [34-36]. Des données récentes utilisant une protéine de fusion entre $\mathrm{Su}(\mathrm{H})$ et le domaine de liaison à l'ADN de Gal4 suggèrent que la protéine $\mathrm{Su}(\mathrm{H})$ agit comme un répresseur transcriptionnel, sans doute à travers le recrutement d'un co-répresseur. La protéine EBNA2 agirait de deux manières: d'une part, en déplaçant le co-répresseur (ce qui explique l'activité d'une molécule EBNA2 dépourvue de domaine d'activation) et, d'autre part, en fournissant un domaine d'activation transcriptionnelle [37].

\section{La protéine $\mathrm{Su}(\mathrm{H})$ et la transduction du signal transmis par Notch}

Chez les mammifères, en plus des homologues déjà cités de Notch, $S u(H)$ et Serrate (codant pour la molécule Jagged citée plus haut), on connaît des homologues potentiels du gène Delta, des gènes du complexe $E(s p l)$, appelés HES-1, 2 et 5 [38], des gènes proneuraux achaete-scute (appelés Mash-1 et -2; [39]), ainsi que du gène deltex (S. ArtavanisTsakonas, communication personnelle), mais aucun homologue du gène Hairless.

Des résultats récents suggèrent un modèle permettant d'expliquer l'activation transcriptionnelle de certains gènes cibles par les formes constitutivement actives de Notch et un mécanisme d'activation de la cascade Notch induite par la fixation de son ligand. Le groupe de R. Kopan (Seattle, WA, USA) a observé que certains mutants de Notch ancrés dans la membrane mais dépourvus de la majorité de la région extracellulaire peuvent être clivés dans différents types cellulaires, libérant la partie intracellulaire qui est alors retrouvée dans le noyau [40]. Sachant que la protéine $\mathrm{Su}(\mathrm{H})$ est capable d'interagir avec les répétitions du motif ankyrine de Notch chez la drosophile, on peut se demander si la protéine $\mathrm{Su}(\mathrm{H})$ et la forme nucléaire de Notch agissent de concert pour activer la transcription de certains gènes cibles. Un de ces 
gènes cibles potentiels est $H E S$ - 1 , qui pourrait représenter un homologue chez les mammifères des gènes du complexe $E(s p l)$, dont la transcription est directement activée par $\mathrm{Su}(\mathrm{H})$ chez la drosophile. Des expériences de cotransfection dans divers types cellulaires ont démontré qu'une forme intracellulaire de Notch est capable d'activer la transcription de HES-1, et que cette activation a lieu par l'intermédiaire de deux sites de fixation pour $\mathrm{Su}(\mathrm{H})$ localisés dans la région proximale du promoteur. D'autre part il a été montré qu'un complexe $\mathrm{Su}(\mathrm{H}) /$ Notch est effectivement capable de se former sur les sites de fixation pour $\mathrm{Su}(\mathrm{H})$ du promoteur de HES-1 [41]. A la suite de ces résultats, il est tentant d'imaginer un modèle dans lequel la fixation d'un ligand de Notch induirait une coupure protéolytique de cette protéine aboutissant à la translocation nucléaire du domaine intracellulaire qui viendrait se lier à la protéine $\mathrm{Su}(\mathrm{H})$ sur les sites de fixation des gènes cibles de la cascade. Dans ce contexte, on peut se demander si la protéine $\mathrm{Su}(\mathrm{H})$ est déjà dans le noyau, fixée sur les régions régulatrices de ces gènes cibles, ou si elle est effectivement retenue dans le cytoplasme par une forme non tronquée de Notch; dans ce dernier cas la fixation du ligand aboutirait à la translocation nucléaire du complexe préformé entre la région intracellulaire de Notch et $\mathrm{Su}(\mathrm{H})$.

Récemment l'inactivation par recombinaison homologue dans la souris du gène $\mathrm{Su}(\mathrm{H})$ a été obtenue par le groupe de T. Honjo [42], et se traduit par le mort des embryons au jour 10. Au jour 9,5, les embryons présentent des défauts tels que microencéphalie, développement anormal du placenta ainsi que du tube neural et somitogenèse défectueuse. Le phénotype au niveau des somites est semblable à celui observé chez les souris dont le gène Notch-1 a été inactivé, bien qu'apparaissant plus tôt. Une coopération des deux protéines au niveau de la somitogenèse pourrait donc être envisagée (voir plus loin).

\section{Inhibition latérale chez les mammifères}

Récemment, plusieurs ligands poten162 férentes espèces de vertébrés (revue: [43]). La protéine Jagged, déjà citée plus haut, semble être l'homologue chez le rat de Serrate plutôt que de Delta, et semble capable d'émettre un signal inhibiteur de différenciation myogénique pour des cellules C2 transfectées par Notch 1. Parallèlement, un homologue de Delta a été isolé chez le Xénope [44], le poulet [45] et la souris [46]. Une approche par PCR a permis le clonage de trois homologues supplémentaires de Delta et de deux de Serrate (D. Henrique, communication personnelle). La multiplicité de ces ligands amène à se demander si toutes ces protéines interagissent avec toutes les protéines Notch ou si une certaine sélectivité existe. Il faut préciser qu'aucune démonstration formelle d'interaction de ces ligands avec une protéine Notch particulière in vivo n'a encore été apportée, à part les expériences dans des cellules transformées par Notch 1 et jagged citées plus haut. En particulier, il est important de noter que jagged n'est pas exprimé au moment de la myogenèse in vivo; en revanche, il est exprimé dans le tube neural où son profil d'expression recouvre celui des trois gènes Notch [30]. En revanche, l'homologue de Delta chez la souris est exprimé dans le mésoderme présomitique et au cours de la condensation somitique [46]. L'analyse de souris ayant subi une inactivation du gène Notch 1 implique ce gène dans la formation de l'épithélium somitique [24, 25]. Une interaction entre Delta et Notch 1 est donc possible au cours de ces événements de différenciation.

En ce qui concerne la neurogenèse, un certain nombre de données suggèrent que des interactions entre Delta et Notch 1 existent. Au cours de la différenciation de la rétine de poulet, il a été montré qu'un signal inhibiteur responsable de la formation d'un petit nombre seulement de cellules différenciées était transmis par Notch 1 [47]. De plus, le mélange de cellules précurseurs et de cellules surexprimant le ligand Delta de drosophile reproduit cette inhibition. Dans le même ordre d'idées, l'injection de l'analogue amphibien de Delta dans des embryons de la même espèce aboutit à une diminution du nombre de neurones. D'autre part Delta est exprimé dans trois régions de chaque côté de la plaque neurale, qui donneront précisément naissance à trois groupes de neurones. Il semble donc que, chez le xénope, Delta et Notch 1 jouent un rôle important en contrôlant le nombre de précurseurs neuroectodermiques qui choisiront un destin neuronal, et que la sélection de ces précurseurs résulte d'un mécanisme d'inhibition latérale.

\section{Conclusion}

En résumé il semble que la voie de signalisation Notch joue un rôle critique en déterminant, probablement par inhibition latérale au sein de groupes de cellules équipotentes, le nombre de cellules qui choisiront un destin neuronal dans la rétine et dans le système nerveux central. Il est probable que ce mécanisme de signalisation par Notch joue un rôle identique au cours d'autres événements de différenciation. Maintenant qu'une bonne partie des acteurs ont été clonés chez les vertébrés supérieurs, une approche biochimique, complémentaire des approches de génétique moléculaire conduites chez la drosophile, devrait permettre de mieux comprendre les interactions entre ces diverses molécules ainsi que les mécanismes détaillés aboutissant à la transmission du signal jusqu'aux gènes cibles

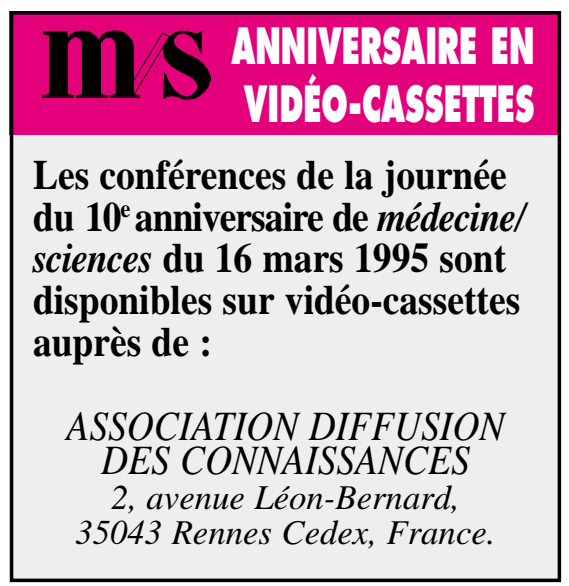

$\mathrm{m} / \mathrm{s} n^{\circ} 2$, vol. 12 , féurier 96 


\section{Summary}

Notch receptor signaling: from flies to mammals

During embryonic development, cellular fate is determined by various types of extracellular signals. A mechanism known as lateral inhibition controls the ability of cells to respond to these various differentiation signals. One of the best studied example is the Notch activation pathway, which controls the differentiation of numerous cell types during development of Drosophila and C. elegans. During embryonic neurogenesis in Drosophila, Notch receptor activation results in transcriptional activation of target genes and inhibition of differentiation. Genetic approaches in flies and worms have defined putative components of this signaling cascade, including factors that may associate with the Notch intracellular domain required for signaling activity. One of them, the $\mathrm{Su}(\mathrm{H})$ protein, is a transcription factor which binds to the regulatory sequences of several Notch target genes. It has been suggested that the intracellular domain of Notch might sequester $\mathrm{Su}(\mathrm{H})$ in the cytoplasm, and that Notch receptor activation might lead to the release of $\mathrm{Su}(\mathrm{H})$ and to its nuclear import. Recent data obtained in vertebrates suggest that the Notch pathway is conserved, both structurally and functionnally. On the basis of these results, a model has been proposed, whereby the intracellular domain of Notch, following a proteolytic event, would translocate to the nucleus and directly activate transcription of its target genes by binding to the $\mathrm{Su}(\mathrm{H})$ protein. 